\title{
Uso de la monitorización de profundidad anestésica
}

\author{
Use of anesthetic deep monitoring
}

\author{
María Fernanda Cortés Barenque, * José Manuel Athié García, ${ }^{\ddagger}$ Jessica Lizeth Morales Gámez,\$ \\ Nancy Verónica Alva Arroyo," Eric Misael Saucedo Moreno"
}

Citar como: Cortés BMF, Athié GJM, Morales GJL, Alva ANV, Saucedo MEM. Uso de la monitorización de profundidad anestésica. Acta Med Grupo Angeles. 2021; 19 (2): 190-194. https://dx.doi.org/10.35366/100441

\section{Resumen}

La monitorización de la relajación neuromuscular y electroencefalográfica se han convertido en herramientas de gran utilidad para un manejo anestésico más seguro. En este estudio se busca conocer la frecuencia de su uso y en qué escenarios son más utilizados. Material y métodos: Estudio observacional, transversal, retrospectivo, con datos obtenidos del registro transanestésico de pacientes sometidos a cirugía de noviembre 2017 a enero 2018. Se realizaron dos subgrupos, uno fue con el uso del índice biespectral (BIS) y otro con tren de cuatro elementos (TOF), buscando identificar algunos factores contribuyentes a la elección entre éstos y la frecuencia de uso. Resultados: Se analizaron 175 registros en los cuales se empleó BIS en 65 pacientes (37.1\%) y TOF en 16 (9\%). AI dividir en dos grupos identificamos diferencias significativas para el uso de TOF frente a BIS si la cirugía era una cirugía mayor (76.5 vs $48.1 \% p=0.037$ ), de igual forma identificamos que en cirugías urgentes se decidió colocar TOF en lugar de BIS 0 vs $17.6 \% p=0.013)$. Conclusiones: Se identificó que el empleo de BIS y TOF está por debajo de $50 \%$, comparado con la estadística mundial, por lo cual se subraya la relevancia del uso rutinario de estas herramientas.

Palabras clave: Tren de cuatro, índice biespectral, monitorización.
* Médico residente de Anestesiología, Alumna de la Facultad Mexicana de Medicina Universidad La Salle.

₹ Director Médico.

§ Médico Anestesiólogo, Algología y Medicina Paliativa.

" Médico Anestesiólogo y Medicina Crítica.

॥ Cirujano General, Maestría en Ciencias Médicas.

Hospital Ángeles Mocel. Ciudad de México. México.

Correspondencia:

María Fernanda Cortés Barenque

Correo electrónico: dra.cortesbarenque@gmail.com

Aceptado: 04-06-2020.

www.medigraphic.com/actamedica

\section{Abstract}

Neuromuscular blockade and electroencephalographic monitoring have become very useful tools for safer anesthetic management. This study seeks to know the frequency of their use and in which scenarios they are most commonly used. Material y methods: An observational, cross-sectional, retrospective study was conducted with data obtained from the trans- anesthetic register of patients undergoing surgery from November 2017 to January 2018. We divided the sample into two subgroups, one was with the use of BIS and the other with TOF, seeking to identify some contributing factors to decide between these. Results: 175 records were analyzed using BIS in 65 patients $(37.1 \%)$ and TOF in $16(9 \%)$. By dividing into two groups we identified significant differences for the use of TOF versus BIS if the surgery was major surgery (76.5 vs $48.1 \% p=0.037$ ), we also identified that in urgent surgeries it was decided to place TOF instead of BIS ( 0 vs $17.6 \% p=$ 0.013). Conclusions: It was reported that the use of BIS and TOF is well below $50 \%$ compared to the global statistics, so we are interested in reminding the relevance of the routinary use of these tools.

Keywords: Train of four, bispectral index, monitoring.

\section{INTRODUCCIÓN}

La mejor forma de abatir la incidencia de complicaciones anestésicas es la evaluación precisa mediante monitoreo electrónico y el manejo perioperatorio integral del paciente quirúrgico. Gracias a los diversos fármacos anestésicos y al monitoreo electrónico avanzado, se logran optimizar las dosis de los agentes anestésicos empleados. ${ }^{1}$ En la actualidad, no hay consenso sobre los parámetros que usan los anestesiólogos para determinar una adecuada reversión del bloqueo neuromuscular.

En un intento por determinar la práctica actual, Grayling realizó un estudio donde realizó una encuesta que abarca 12 departamentos de anestesia en todo el Reino Unido; 
se pidió a las personas que proporcionaran detalles sobre el uso de los monitores disponibles o, en su defecto, que enumeraran los parámetros clínicos que consideraban que ofrecían la mejor orientación en cuanto a la adecuada recuperación del bloqueo neuromuscular. No hubo consenso entre los anestesiólogos sobre los signos clínicos más confiables de recuperación del bloqueo neuromuscular. ${ }^{2}$

De igual forma, creemos que existe una aparente confusión general entre los médicos sobre el mejor método para confirmar la recuperación del bloqueo neuromuscular. Quizá esto se deba a que no se confía lo suficiente en el uso de monitores cuantitativos o una falta de claridad en las guías con respecto al monitoreo de la función neuromuscular. El propósito de este estudio es conocer en nuestro medio la frecuencia de aplicaciones de elementos para vigilancia de profundidad anestésica como el índice biespectral (BIS por sus siglas en inglés) y el tren de cuatro estímulos (TOF por sus siglas en inglés).

\section{MATERIAL Y MÉTODOS}

Realizamos un estudio observacional, transversal, retrospectivo, en un hospital privado de la Ciudad de México, con datos obtenidos de expedientes. Incluimos todos los casos que fueron operados de noviembre de 2017 a enero de 2018, mayores de 18 años, bajo anestesia general; excluimos a los que no tenían datos completos en el expediente para el estudio. Analizamos las siguientes variables: estado físico según la Asociación Americana de Anestesiología (ASA), tipo de anestesia empleada, tiempo en minutos de la anestesia, tipo de cirugía (mayor o menor), diagnóstico quirúrgico y clínico, y el tipo de monitorización neurológica (TOF o BIS) para vigilancia de la profundidad anestésica y relajación neuromuscular.

Realizamos un subanálisis dividiendo la muestra en dos grupos. El grupo 1 representa a los pacientes que fueron sometidos a neurovigilancia con BIS; y el grupo 2 representa a los pacientes en quienes se utilizó TOF, con la intención de identificar algún factor que contribuya a la elección entre BIS y TOF. Hicimos un análisis descriptivo de los datos. Para variables cuantitativas con distribuciones no paramétricas utilizamos prueba $U$ de Mann-Whitney, fueron expresadas como medianas, mínimos y máximos. La prueba $\chi^{2}$ o Fisher la utilizamos para datos categóricos. Consideramos un valor menor a 0.05 para una $p$ significativa con IC del 95\%. Los datos se analizaron utilizando el software SPSS (versión 20.0 para Windows).

\section{RESULTADOS}

Estudiamos una muestra de 175 expedientes que cumplieron con los criterios de selección, la edad media fue de $47.5 \pm 16.7$ años, sexo femenino 95 (54\%), rango de peso normal 83 pacientes (47.4\%), sobrepeso 63 pacientes (36\%). ASA II 99 pacientes (56.6\%), se hizo cirugía electiva en 164 casos (94\%) y emplearon videolaringoscopio en 64 (37\%). Las demás características se muestran en la Tabla 1. El tiempo empleado en la anestesia promedio de estas cirugías fue $169.04 \pm 68.22$ minutos. Se empleó anestesia general balanceada en 157 pacientes (89.7\%), el halogenado más empleado fue sevoflurano en 130 casos (85\%) y anestesia total intravenosa (TIVA) en 18 pacientes (10.3\%). Se empleó BIS en 65 sujetos (37.1\%), y TOF en 16 pacientes $(9 \%), 42.9 \%$ de las cirugías realizadas fueron cirugías mayores, donde se empleó con mayor frecuencia el TOF en el $76.5 \%$ de los casos (Tablas 1 y 2).

Realizamos un subanálisis al dividir en dos grupos de acuerdo con la monitorización utilizada. Identificamos

Tabla 1: Variables generales de la muestra. $\mathrm{N}=175$

\begin{tabular}{|c|c|}
\hline & $\mathrm{n}(\%)$ \\
\hline Edad* & $47.50 \pm 16.732$ \\
\hline Peso* & $73.81 \pm 10.68$ \\
\hline Sobrepeso & $63(36.0)$ \\
\hline \multicolumn{2}{|l|}{ Obesidad (grado) } \\
\hline 1 & $27(15.4)$ \\
\hline II & $1(0.6)$ \\
\hline III & $1(0.6)$ \\
\hline Talla* & $1.66 \pm 0.7649$ \\
\hline \multicolumn{2}{|l|}{ ASA } \\
\hline I & $76(43.2)$ \\
\hline II & 99 (56.6) \\
\hline \multicolumn{2}{|l|}{ Tipo de anestesia } \\
\hline General balanceada & $157(89.7)$ \\
\hline TIVA & $18(10.3)$ \\
\hline \multicolumn{2}{|l|}{ Halogenado empleado } \\
\hline Sevoflurano & $130(85.0)$ \\
\hline Desfluorano & $45(15.0)$ \\
\hline Tiempo de anestesia* & $169.04 \pm 68.22$ \\
\hline Cirugía mayor & $82(46.9)$ \\
\hline \multicolumn{2}{|l|}{ Tipo de cirugía } \\
\hline Urgencia & $11(6.3)$ \\
\hline Electiva & $164(94)$ \\
\hline BIS & $65(37.1 \%)$ \\
\hline Rango de BIS* & $14.73 \pm 20.31$ \\
\hline TOF & $16(9.1)$ \\
\hline \multicolumn{2}{|l|}{ Neurovigilancia } \\
\hline Ninguno & $106(60.6)$ \\
\hline TOF & $15(9.7)$ \\
\hline BIS & $52(29.7)$ \\
\hline
\end{tabular}

ASA = Asociación Americana de Anestesiología, TIVA = anestesia total intravenosa, BIS = índice biespectral, TOF = tren de cuatro.

* Los datos indican la media \pm desviación estándar. 
Tabla 2: Comparación entre grupos de acuerdo a tipo de neurovigilancia.

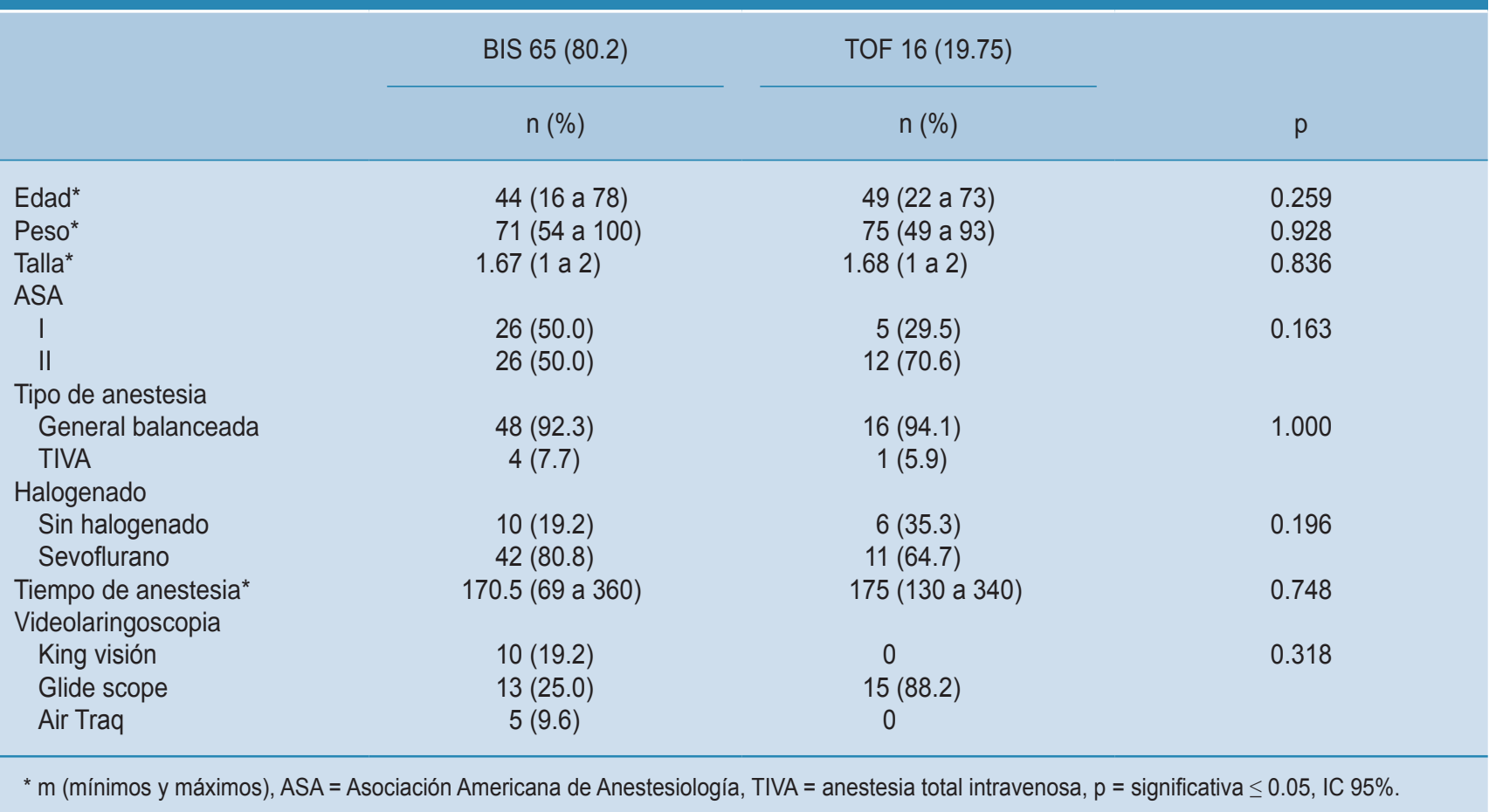

diferencias estadística y clínicamente significativas para el uso de TOF frente a BIS si la cirugía se consideraba mayor (76.5 vs $48.1 \% \mathrm{p}=0.037$ ), de igual forma, identificamos que en cirugías urgentes se decidió colocar TOF en lugar de BIS (0 vs $17.6 \% \mathrm{p}=0.013$ ) (Tabla 2).

Llama la atención que el ASA y el tipo de anestesia empleada no presentaron diferencias significativas entre uno y otro grupo, $p=0.163$ y $p=1.000$ respectivamente.

\section{DISCUSIÓN}

Para monitorizar la profundidad anestésica se hace un análisis de la señal de la electroencefalografía (EEG). Monitorizarla tiene como primer objetivo ajustar en tiempo real las cantidades del agente anestésico administrado al paciente de acuerdo con sus requerimientos. La profundidad de la anestesia se fundamenta en los efectos depresores sobre el sistema nervioso autónomo en respuesta a las concentraciones progresivamente más elevadas del anestésico inhalatorio o intravenoso; el nivel de anestesia tiene que ser lo suficientemente profundo como para impedir la conciencia del paciente. Muchos anestesiólogos se basan en la estimulación quirúrgica que, al no provocar movimientos ni variaciones hemodinámicas, asumen que una profundidad anestésica es adecuada. ${ }^{3}$ Estos parámetros clínicos pueden fallar y distorsionar la medida de la profundidad anestésica, dando origen en ocasiones al despertar intraoperatorio. De ahí el interés de incorporar parámetros derivados del EEG calculados en tiempo real en la monitorización del grado de hipnosis durante la anestesia. ${ }^{4}$ El monitor BIS mide el índice biespectral (BIS) que es el resultado de la integración de varios subparámetros derivados de distintos análisis del EEG. En su algoritmo se incluyen análisis en el dominio del tiempo, frecuencia y biespectral. ${ }^{5}$ La combinación de estos subparámetros utilizando un algoritmo basado en la observación clínica produce el valor BIS. Este monitor entrega además un índice de actividad electromiográfica (EMG) y de tasa de supresión del EEG. ${ }^{6}$ Entre las ventajas de su uso está la titulación anestésica basada en la actividad cerebral, con la cual se disminuye la incidencia de despertar intraoperatorio y el consumo anestésico, lo que conlleva una rápida recuperación.

Este índice puede fluctuar entre 100 (despierto) y 0 (actividad cerebral mínima). Los índices de 0-100 representan valores promedios de los últimos 15 a 30 segundos de señal, que van siendo entregados en tiempo real (aproximadamente cada segundo). Los valores recomendados para una anestesia quirúrgica están entre 40 y $60 .^{8}$

Los valores de BIS para control de sedación y anestesia son: 1) > 90 despierto, memoria intacta; 2) 65-85 sedación; 3) 40-60 anestesia general; 4) < 40 anestesia excesivamente profunda; y 5) tendencia a la isoelectricidad. ${ }^{9}$ 
La Sociedad Americana de Medicina Perioperatoria realizó un consenso publicado en 2020 en donde analizan el papel de la neuromonitorización transquirúrgica y hacen las siguientes recomendaciones:

1. Uso del EEG para guiar el manejo anestésico, con un grado de evidencia $\mathrm{D}$.

2. Capacitación de los médicos para la interpretación del electroencefalograma al momento de usar esta tecnología en el manejo anestésico (grado de evidencia $C$, recomendación fuerte). Se realizó un estudio de 40 anestesiólogos, en donde la mayoría fueron capaces de reconocer los estados en los cambios en el electroencefalograma relacionadas con los estados anestésicos después de un tutorial de 15 minutos; de igual forma, se dio entrenamiento de 45 minutos a un grupo de médicos en donde lograron leer el electroencefalograma dándole un puntaje de BIS similar al valor que generaba la máquina. En la actualidad, existen tutoriales disponibles para lograr esta capacitación en www.icetap.org.

3. Recomiendan el uso de monitoreo del electroencefalograma para disminuir el riesgo de despertar intraoperatorio (grados de evidencia C, recomendación fuerte). En procedimientos bajo anestesia general balanceada no hay una diferencia significativa si se compara el uso de monitor electroencefalográfico vs la medición convencional de la concentración de gases al final de la expiración (ETAG), combinado con un monitoreo anestésico con alarmas del monitor prendidas a volumen adecuado; sin embargo, en anestesia total endovenosa sí se han encontrado diferencias significativas, presentando menor incidencia de despertar intraoperatorio con el uso de BIS hasta en un 51.9\%.

4. En cuanto a la relación de monitoreo EEG con la incidencia de delirium postoperatorio en adultos mayores, concluyen que aún no se cuenta con evidencia suficiente al respecto. ${ }^{10}$

Por otra parte, se ha planteado que la monitorización del nivel de bloqueo neuromuscular mediante la técnica del tren de cuatro estímulos (TOF) puede ser útil. Ha permanecido como el método más útil para la evaluación de la función neuromuscular durante más de 40 años, se basa en la observación de que el aumento en la frecuencia de estimulación produce fatiga muscular o debilitamiento. ${ }^{11}$ Consiste en la aplicación de cuatro estímulos eléctricos supramáximos con un intervalo entre ellos de $0.5 \mathrm{~s}$ de 2 Hertz $(\mathrm{Hz})$. En ausencia de bloqueo neuromuscular, se obtienen cuatro contracciones iguales en el músculo dependiente del nervio estimulado, cuya evaluación se obtiene al dividir la amplitud de la cuarta respuesta en contraste con la primera, obteniéndose de tal división el coeficiente TOF, el cual va de 0 a $100 \%$ y es inversamente proporcional al grado de bloqueo neuromuscular. ${ }^{12,13} \mathrm{El}$ TOF ha sido recomendado en la práctica clínica debido a que es el test que mide exclusivamente la función neuromuscular, capaz de dar información aunque no se haya obtenido un valor control previo, es fácil de usar y puede ser utilizado de forma repetitiva. ${ }^{14}$ Es de relevancia dentro de este marco que en la mayoría de la bibliografía se sugiere el uso de los signos clínicos como herramienta para evaluar el grado de bloqueo neuromuscular (ordenar apertura de ojos, apretar la mano, levantar la cabeza por cinco segundos, sacar la lengua, depresor lingüal y parámetros respiratorios); sin embargo, son poco sensibles y específicos.

Grayling M en 2007 estudió si existía un consenso entre los anestesiólogos sobre los signos clínicos más confiables de recuperación del bloqueo neuromuscular. Identificaron que $62 \%$ de los pacientes no utilizaron la monitorización anestésica. ${ }^{2}$ De igual forma, pudimos identificar en nuestro estudio que en menos del $45 \%$ de los pacientes sometidos a cirugía se utiliza un monitoreo de profundidad anestésica o bloqueo neuromuscular (BIS o TOF). Una observación relevante es que la Norma Oficial Mexicana para la práctica de la anestesiología sólo sugiere el uso de este tipo de monitoreo no incluye al monitoreo de la trasmisión neuromuscular, ni de electroencefalografía como monitores esenciales en los actos anestésicos. ${ }^{15}$

Castellon-Larios y su grupo de trabajo realizaron una revisión sistemática que incluía 2,526 pacientes, en el que estudiaron la monitorización biespectral y el despertar intraoperatorio. Concluyendo que las interacciones de los fármacos utilizados en la anestesia deben proporcionar al paciente una hipnosis adecuada, además de una analgesia suficiente en conjunto con una buena relajación neuromuscular, evaluada por un monitor anestésico, con la intención de evitar un despertar intraoperatorio. ${ }^{16}$

Una de las debilidades de nuestro trabajo es sin duda la cantidad de pacientes estudiados. Sin embargo, obtuvimos diferencias clínicas y estadísticamente significativas para poder intuir que una de las razones por las cuales no utilizamos la monitorización anestésica es debido a la falta de conocimiento de los diferentes tipos de monitores, además de la falta de guías que estandaricen la monitorización de los pacientes durante cirugías, independientemente de que éstas sean urgencias o cirugías mayores. De igual forma, logramos hacer del conocimiento a la comunidad médica sobre la importancia de su uso, de ahí que surgen inquietudes donde se investigue el despertar anestésico y otras líneas de investigación enfocadas en conocer las complicaciones por la falta de empleo de instrumentos de monitorización anestésica. 


\section{CONCLUSIÓN}

En la práctica privada identificamos que el empleo de BIS y TOF como monitorización de profundidad anestésica y de bloqueo neuromuscular está muy por debajo del 50\%, comparado con la estadística mundial, sin duda alguna debe ser utilizado con mayor frecuencia para la seguridad del paciente sometido a procedimientos anestésicos. A pesar de no estar en la NOM 006 para la práctica de la anestesiología como monitoreo obligatorio, sí se encuentra sugerido, por lo cual si se lleva a cabo un acto quirúrgico y se cuenta con el equipo para neuromonitoreo y monitoreo de bloqueo neuromuscular es importante utilizarlo para mejorar el manejo anestésico y la seguridad del paciente. De igual manera, la capacitación continua y el compromiso de los médicos anestesiólogos resulta vital para conocer los equipos de monitoreo en el mercado y su adecuada interpretación.

\section{REFERENCIAS}

1. American Geriatrics Society; John A. Hartford Foundation. A statement of principles: toward improved care of older patients in surgical and medical specialties. Arch Phys Med Rehabil. 2002; 83 (9): 1317-1319.

2. Grayling M, Sweeney BP. Recovery from neuromuscular blockade: a survey of practice. Anaesthesia. 2007; 62 (8): 806-809.

3. Mashour GA, Wang LY, Turner CR, Vandervest JC, Shanks A, Tremper KK. A retrospective study of intraoperative awareness with methodological implications. Anesth Analg. 2009; 108 (2): 521-526.

4. Gallardo HA, Hernández PAL, Sánchez LJA, Ordoñez EG, Islas AS, Revilla MC. Monitores de profundidad anestésica. Rev Mex Anestesiol. 2016; 39: 201-204.
5. Rodríguez NR, Miranda Cl, García AJ, Benevides FS, Barbosa ROYG, Abitbol MDG. Índice bispectral y otros parámetros procesados del electroencefalograma: una actualización. Rev Bras Anestesiol. 2012; 62 (1): 105-117.

6. Sandin RH, Enlund G, Samuelsson P, Lennmarken C. Awareness during anaesthesia: a prospective case study. Lancet. 2000; 355: 707-711.

7. Davidson AJ, Huang GH, Czarnecki C, Gibson MA, Stewart SA, Jamsen $\mathrm{K}$ et al. Awareness during anaesthesia in children: a prospective cohort study. Anesth Analg. 2005; 100: 653-661.

8. Higuera-Medina LE. ¿Es recomendable el uso del índice biespectral en todo paciente bajo anestesia? Rev Mex Anest. 2010; 33: S64-S66.

9. Leslie K, Myles PS, Forbes A, Chan MTV. The effect of bispectral index monitoring on long-term survival in the B-aware trial. Anesth Analg. 2010; 110: 816-822.

10. Chan MTV, Hedrick TL, Egan TD, García PS, Koch S, Purdon PL et al. American Society for Enhanced Recovery and perioperative quality initiative joint consensus statement on the role of neuromonitoring in perioperative outcomes: electroencephalography. Anesth Analg. 2020; 130 (5): 1278-1291.

11. Shepherd J, Jones J, Frampton G, Bryant J, Baxter L, Cooper K. Clinical effectiveness and cost-effectiveness of depth of anaesthesia monitoring (E-Entropy, Bispectral Index and Narcotrend): a systematic review and economic evaluation. Health Technol Assess. 2013; 17 (34): 1-264.

12. Brice DD, Hetherington RR, Utting JE. A simple study of awareness and dreaming during anaesthesia. Br J Anaesth. 1970; 42 (6): 535-542.

13. Liu SS. Effects of bispectral index monitoring on ambulatory anesthesia: a meta-analysis of randomized controlled trials and a cost analysis. Anesthesiology. 2004; 101 (2): 311-315.

14. Baumann, MH, McAlpin W, Brown K, Patel P, Ahmad I, Stewart $\mathrm{R}$, Petrini M. A prospective randomized comparison of train-offour monitoring and clinical assessment during continuous ICU cisatracurium paralysis. Chest. 2004; 126 (4): 1267-1273.

15. Norma Oficial Mexicana NOM-006-SSA3-2011, para la práctica de la anestesiología.

16. Castellon-Larios K, Rosero B, María Nino-de Mejía C, Bergese S. Uso de monitorización cerebral para el despertar intraoperatorio. Rev Colomb Anestesiol. 2016; 44: 23-29. 